\title{
The knowledge and perceptions regarding antibiotic stewardship of the interns rotating at the Bloemfontein Academic Complex
}

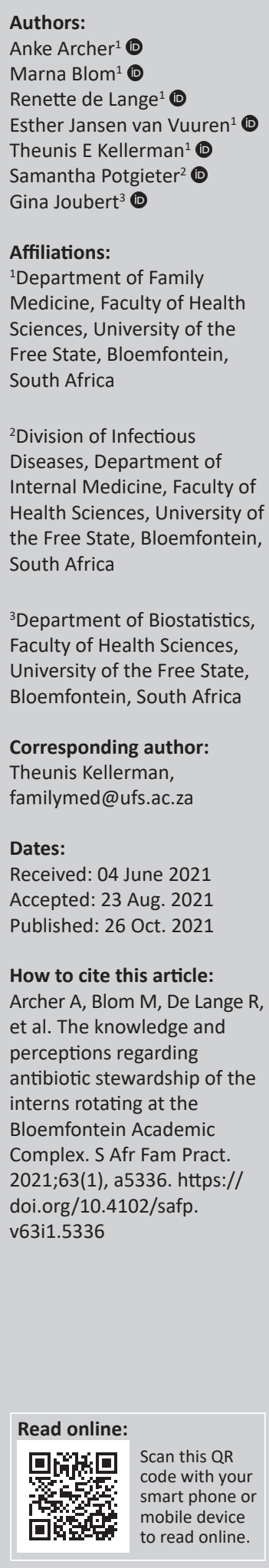

Background: Antibiotic resistance (ABR) is a global problem with the overuse of antibiotics accelerating this process. Antibiotic stewardship aims to optimise antibiotic treatment to enable cost-effective therapy and improve patients' outcome whilst limiting ABR. The study aimed to evaluate intern medical doctors' knowledge and perceptions about antibiotic stewardship and their perceptions regarding education on relevant topics.

Methods: This was a cross-sectional study on interns rotating at Bloemfontein Academic Complex. An anonymous, self-administered questionnaire was completed. The questionnaire recorded demographic information, perception and knowledge of antibiotic stewardship, and the quality of education as perceived by the interns.

Results: Of the 120 possible participants, 92 (76.7\%) responded to all or part of the questionnaire. The median age of the respondents was 25 years, and $56.7 \%$ of the respondents were female. The mean score for the knowledge-based case scenarios was 5.4 out of 10 . Only $4.4 \%$ participants could manage a drip site infection correctly, whilst $18.5 \%$ could treat Escherichia coli (E. coli) bacteraemia. The interns perceived that they have a lack of training and preparedness in certain areas of prescribing antibiotics. Though $77.2 \%$ of the interns had received education on starting antibiotic treatment, $29.3 \%$ claimed to be unsure when to start antibiotic therapy. Interns indicated that formal lectures $(81.3 \%)$ and bedside tutorials $(86.7 \%)$ have a high educational value.

Conclusion: Intern medical doctors do not have sufficient knowledge to establish antibiotic stewardship but have a desire for improvement. The results identified specific areas where better antibiotic training is required.

Keywords: antibiotic resistance; medical interns; knowledge; perception; education.

\section{Introduction}

Antibiotic resistance (ABR) is a worldwide problem with the overuse of antimicrobial substances accelerating this process. ${ }^{1,2}$ Prolongation of antibiotic treatment also promotes ABR, which can lead to extended infection periods, morbidity and mortality. ${ }^{3,4}$

The treatment of patients with resistant infections is more expensive than treatment and care for patients with non-resistant infections, with lengthier hospital stays and the need for more intensive care. In a healthcare system that is already struggling to cope with the burden of disease, the prolongation of hospital stay because of ABR will be devastating on a country's healthcare system. ${ }^{3,4}$

Antibiotic stewardship can be defined as a set of strategies that encourage the practice of giving the correct antibiotics and dosage via the correct application route for the right amount of time. ${ }^{2}$ Antibiotic stewardship aims to optimise antibiotic treatment to enable cost-effective therapy and improve patients' outcome whilst limiting ABR. ${ }^{5}$

The World Health Organization (WHO) has constructed an action plan regarding antimicrobial resistance that was presented at the May 2015 World Health Assembly. The action plan mainly consists of spreading awareness about ABR, improving knowledge through research, minimising infection by improving prevention strategies and increasing investment in interventions. ${ }^{6}$ 
Antibiotic stewardship education is crucial to provide foundational knowledge regarding antibiotics and ABR, making the correct diagnosis and infection management and appropriate prescribing. Education on appropriate prescribing for healthcare providers has shown to improve other antibiotic stewardship interventions. ${ }^{7}$ Improved communication skills will also help to convey this information to patients. ${ }^{7}$

In South Africa, the primary obstacle in antimicrobial stewardship programmes in almost all public and private health facilities has been insufficient resources and expertise about infectious diseases. ${ }^{8}$

Studies in Europe, ${ }^{9}$ Australia ${ }^{10}$ and South Africa ${ }^{11}$ have found that medical students have limited knowledge and confidence regarding antibiotic prescription. Similarly, studies in the United Kingdom ${ }^{12,13}$ have highlighted the needs and challenges faced by junior doctors (who frequently are the prescribers of antibiotics) regarding antibiotic prescribing.

This study aimed to determine the knowledge and perceptions of intern medical doctors in Bloemfontein regarding antibiotic prescribing and stewardship and their perceptions on the usefulness of different educational activities for learning about antibiotic-related topics.

\section{Materials and methods \\ Study design, population and sampling}

This was a cross-sectional study. The target population included all 120 first- and second-year intern medical doctors rotating at the Bloemfontein Academic Complex through eight departments from August 2017 to October 2017. The departments included: Family Medicine, Surgery, Anaesthesiology, Paediatrics, Orthopaedics, Obstetrics and Gynaecology, Internal Medicine, and Psychiatry.

\section{Data collection and measurements}

An anonymous, self-administered questionnaire in English was handed out to interns at the end of a formal lecture after the study, and its aim was explained by the researchers. Lecturers assisted in questionnaire distribution and collection, ensuring that questionnaires were completed individually and not as group work. Completed questionnaires were handed in at the door.

Permission was granted by Dr Sean Wasserman (University of Cape Town) to use a questionnaire composed by researchers from the University of Cape Town who conducted a study on antibiotic stewardship on final-year medical students at three medical schools (University of the Witwatersrand, University of Cape Town and University of the Free State [UFS]). ${ }^{11}$ Some of the questions were altered to accommodate the difference between the study populations of the two studies.

The questionnaire consisted of sections on demographics (medical school attended, gender, age, previous qualifications), knowledge (self-reported awareness of antibiotic guidelines and the term antibiotic stewardship, 10 clinical case scenarios with five options to choose the correct answer from) and perceptions regarding antibiotics, their causes and outcomes, the usefulness of various educational activities to learn about antibiotic classes, antibiotic prescribing and resistance.

\section{Data analysis}

Results were summarised by frequencies and percentages (categorical variables) and means, medians and interquartile ranges (IQRs) (numerical variables). The analysis was done by the Department of Biostatistics, Faculty of Health Sciences, UFS.

\section{Pilot study}

A pilot study was conducted mainly to determine the amount of time needed to complete the questionnaire. The pilot study consisted of a group of 11 fifth-year medical students from UFS, who were selected on a single day as a convenient sample at National District Hospital. Questionnaires were handed out to the students after a formal lecture and were collected as the students left the venue. No changes were made to the questionnaire.

\section{Ethical considerations}

The protocol was approved by the Health Sciences Research Ethics Committee, UFS (HSREC-S 13/2017). Permission was obtained from the Free State Department of Health to include intern medical doctors in this study.

\section{Results}

Of the 120 interns, $92(76.7 \%)$ responded to all or part of the questionnaire. The 28 interns who did not respond consisted of 11 who were on leave, 1 who refused and 16 who were unavailable during the time of data collection. The median age of the respondents was 25 years (IQR: 24-26 years) and $56.7 \%$ of the respondents were female. All South African medical schools were represented amongst the respondents with the UFS representing the largest percentage (34.1\%). Five respondents indicated that they received their medical training outside South Africa.

\section{Knowledge}

According to $59.3 \%$ (51 of 86 ) of the respondents, the hospitals where they work have antibiotic guidelines. The majority of respondents $(81.5 \%, 75$ of 92$)$ indicated that they have used or consulted antibiotic guidelines.

Half $(54.4 \%, 50$ of 92) of the respondents reported being very familiar with the term 'antibiotic stewardship' and 34.8\% reported being familiar, that is, they have heard the term mentioned in lectures or tutorials, but it was not explained further. 
The mean score for the knowledge-based case scenarios was 5.4 out of 10 . Only $6.5 \%$ of the respondents had a score of 8 or more out of 10 and $77.2 \%$ had a score of 5 or more out of 10 .

Most of the respondents $(76.1 \%, n=70)$ knew what antibiotic to prescribe when managing an uncomplicated urinary tract infection. However, only $29.4 \%(n=27)$ knew what antibiotic to prescribe for methicillin-sensitive Staphylococcus aureus.

The majority of the respondents $(83.7 \%, n=77)$ knew how to assess the severity and management of community-acquired pneumonia. Less than two-thirds $(62.0 \%, n=57)$ of the interns knew how to interpret an antibiogram and de-escalate antibiotic treatment, and the same percentage knew how to manage an upper respiratory tract infection.

The correct management of a drip site infection was only identified by $4.4 \%(n=4)$ of the respondents. Half $(47.8 \%$, $n=44)$ knew how to correctly assess the severity and management of a Clostridium difficile infection.

The correct antimicrobial selection for extended-spectrum beta-lactamases (ESBL)-positive Escherichia coli bacteraemia was made by $18.5 \%(n=17)$ of the respondents. Perioperative antibiotic prophylaxis duration and risks of excessive use were assessed correctly by $73.9 \%(n=68)$ of the respondents. The majority of respondents $(87.0 \%$, $n=80$ ) knew how to manage the poor response to empiric antibiotic therapy.

\section{Perceptions regarding antibiotic stewardship}

Respondents' answers regarding perceptions relating to antibiotic prescribing indicated that the majority were aware of $A B R$, its causes and outcomes and would like more education on ABR and appropriate use of antibiotics (Table 1). Only $50.0 \%$ agreed that lack of hand disinfection by healthcare workers causes the spread of ABR, and 29.4\% chose the option neutral for this question. Although more than $50 \%$ disagreed with the statement that new antibiotics to deal with $A B R$ are available, a quarter chose the option neutral.

\section{Perceptions regarding preparedness in antibiotic prescribing}

As summarised in Table 2, most of the respondents reported that their medical school training prepared them well for making an accurate diagnosis (84.8\%) and knowing when to start antibiotic treatment $(70.7 \%)$. Only $17.6 \%$ felt that they were prepared on how to interpret antibiograms, whereas more than half $(57.1 \%)$ rated their preparedness as poor or very poor. On most of these statements, between a quarter and a third of respondents chose the neutral option, with the highest percentage in this category being for the statement regarding knowledge of dosing and duration (42.4\%).

\section{Perceptions regarding education on relevant topics}

As indicated in Table 3, formal bedside tutorials were the educational method chosen by the largest percentage of respondents as being useful. More than $80 \%$ of the respondents

TABLE 1: Summary of the respondents' perceptions regarding antibiotic prescribing.

\begin{tabular}{|c|c|c|c|c|c|c|}
\hline \multirow[t]{2}{*}{$\overline{\text { Statement }}$} & \multicolumn{2}{|c|}{ Disagree/strongly disagree } & \multicolumn{2}{|c|}{ Neutral } & \multicolumn{2}{|c|}{ Agree/strongly agree } \\
\hline & $n$ & $\%$ & $n$ & $\%$ & $n$ & $\%$ \\
\hline Antibiotics are overused in South Africa & 3 & 3.3 & 8 & 8.7 & 81 & 88.0 \\
\hline Antibiotics are overused at the hospitals where I have rotated & 10 & 10.9 & 12 & 13.0 & 70 & 76.1 \\
\hline Antibiotic resistance is a significant problem in South Africa & 3 & 3.3 & 10 & 10.9 & 79 & 85.9 \\
\hline Antibiotic resistance is a significant problem at the hospitals where I have rotated & 5 & 5.4 & 29 & 31.5 & 58 & 63.0 \\
\hline New antibiotics are available to deal with the problem & 54 & 58.7 & 24 & 26.1 & 14 & 15.2 \\
\hline Inappropriate use of antibiotics causes antibiotic resistance & 0 & 0.0 & 2 & 2.2 & 90 & 97.8 \\
\hline Better use of antibiotics will reduce levels of antibiotic resistance & 1 & 1.1 & 5 & 5.4 & 86 & 93.5 \\
\hline $\begin{array}{l}\text { Use of broad-spectrum antibiotics when equally effective narrower spectrum antibiotics } \\
\text { are available can increase antibiotic resistance }\end{array}$ & 6 & 6.5 & 5 & 5.4 & 81 & 88.0 \\
\hline Lack of hand disinfection by healthcare workers causes the spread of antibiotic resistance & 19 & 20.7 & 27 & 29.4 & 46 & 50.0 \\
\hline Inappropriate use of antibiotics is directly harmful to patients & 3 & 3.3 & 15 & 16.3 & 74 & 80.4 \\
\hline A good knowledge of antibiotics is important to my work as doctor & 0 & 0.0 & 1 & 1.1 & 91 & 98.9 \\
\hline I would like more education on antibiotic resistance & 2 & 2.2 & 8 & 8.7 & 82 & 89.1 \\
\hline I would like more education on the appropriate use of antibiotics & 1 & 1.1 & 10 & 10.9 & 81 & 88.1 \\
\hline
\end{tabular}

TABLE 2: Interns' self-reported perceptions regarding their preparedness to prescribe antibiotics $(n=92)$.

\begin{tabular}{|c|c|c|c|c|c|c|}
\hline \multirow[t]{2}{*}{ Statement } & \multicolumn{2}{|c|}{ Good/very good } & \multicolumn{2}{|c|}{ Neutral } & \multicolumn{2}{|c|}{ Poor/very poor } \\
\hline & $n$ & $\%$ & $n$ & $\%$ & $n$ & $\%$ \\
\hline Making accurate diagnosis & 78 & 84.8 & 13 & 14.1 & 1 & 1.1 \\
\hline Knowing when to start antibiotics & 65 & 70.7 & 25 & 27.2 & 2 & 2.2 \\
\hline Choosing the correct antibiotic & 51 & 55.4 & 35 & 38.0 & 6 & 6.5 \\
\hline Knowledge of dosing and duration & 40 & 43.5 & 39 & 42.4 & 13 & 14.1 \\
\hline How to de-escalate to narrower spectrum & 35 & 38.0 & 33 & 35.9 & 24 & 26.1 \\
\hline How and when to transition from intravenous to oral & 47 & 51.1 & 27 & 29.4 & 18 & 19.6 \\
\hline How to interpret antibiograms $(n=91)$ & 16 & 17.6 & 23 & 25.3 & 52 & 57.1 \\
\hline Understanding spectrum of activity & 48 & 52.2 & 28 & 30.4 & 16 & 17.4 \\
\hline Understanding basic mechanisms of resistance & 55 & 59.8 & 30 & 32.6 & 7 & 7.6 \\
\hline
\end{tabular}


TABLE 3: Perceptions of the respondents regarding the usefulness of different educational methods in the medical curriculum.

\begin{tabular}{|c|c|c|c|c|c|c|}
\hline \multirow[t]{2}{*}{ Variable } & \multicolumn{2}{|c|}{ Not useful at all/not useful } & \multicolumn{2}{|c|}{ Neutral } & \multicolumn{2}{|c|}{ Useful/very useful } \\
\hline & $n$ & $\%$ & $n$ & $\%$ & $n$ & $\%$ \\
\hline \multicolumn{7}{|c|}{ Antibiotic classes and spectrums of activity } \\
\hline Formal lectures $(n=91)$ & 4 & 4.4 & 14 & 15.4 & 73 & 80.2 \\
\hline Formal bedside tutorials ( $n=91$ ) & 3 & 3.3 & 8 & 8.8 & 80 & 87.9 \\
\hline Consultant ward rounds ( $n=91)$ & 5 & 5.0 & 10 & 11.0 & 76 & 83.5 \\
\hline Registrar ward rounds $(n=92)$ & 12 & 13.0 & 18 & 19.6 & 62 & 67.4 \\
\hline Problem-based learning $(n=91)$ & 6 & 6.6 & 9 & 9.9 & 76 & 83.5 \\
\hline \multicolumn{7}{|c|}{ Antibiotic prescribing and resistance } \\
\hline Formal lectures $(n=91)$ & 5 & 5.5 & 12 & 13.2 & 74 & 81.3 \\
\hline Formal bedside tutorials ( $n=90$ ) & 2 & 2.2 & 10 & 11.1 & 78 & 86.7 \\
\hline Consultant ward rounds ( $n=91$ ) & 5 & 5.5 & 10 & 11.0 & 76 & 83.5 \\
\hline Registrar ward rounds ( $n=92$ ) & 12 & 13.0 & 20 & 21.7 & 60 & 65.2 \\
\hline Problem-based learning $(n=90)$ & 6 & 6.7 & 12 & 13.3 & 72 & 80.0 \\
\hline
\end{tabular}

also rated formal lectures, consultant ward rounds and problem-based learning as useful when it comes to antibiotic classes and spectrums of activity as well as antibiotic prescribing and resistance. Respondents found consultant ward rounds more useful than registrar ward rounds.

The majority of respondents had attended lectures or tutorials for the following: diagnosis of infection $(85.9 \%, n=79$ ), indications for starting antibiotics $(77.2 \%, n=71)$, dosing and duration of antibiotic therapy for specific infection $(76.1 \%$, $n=70)$, indications for intravenous antibiotics $(78.3 \%, n=72)$, rational use of antibiotics in general $(78.3 \%, n=72)$ and basic principles of infection prevention and control $(85.9 \%, n=79)$.

The respondents indicated that a local handbook or guidelines on treating common infections $(78.3 \%, n=72)$, more applications for smart devices $(58.7 \%, n=54)$ and more exposure to infectious diseases specialists $(53.3 \%, n=49)$ were ways to prepare newly qualified doctors to prescribe antibiotics. Less than half suggested more formal bedside tutorials $(41.3 \%, n=38)$, more formal lectures $(31.5 \%, n=29)$ and more time in microbiology $(25.0 \%, n=23)$. Only $10.9 \%$ $(n=10)$ selected 'more computer-based tutorials' as a preparation method.

\section{Discussion}

Of the 120 possible candidates, $76.7 \%$ responded to all or part of the questionnaire. This is considered a good response rate and was in line with the $80 \%$ reported for UFS and the $75 \%$ reported for the University of Cape Town in the study conducted on the final-year medical students. ${ }^{11}$

\section{Knowledge}

In the study done on final-year medical students, ${ }^{11}$ which used the same clinical case scenarios, the mean correct score was $50.0 \%$. There is thus little difference in knowledge between final-year medical students and intern medical doctors (5.4 out of 10). It can be assumed that most of the interns' knowledge is acquired during training in medical school.

From the knowledge questions, it was clear that interns knew what antibiotics to prescribe for a urinary tract infection and how to assess the severity and management of communityacquired pneumonia. This is in accordance with the South African final-year medical student study. ${ }^{11}$ This can be expected as urinary tract infections and pneumonia are relatively common diseases in South Africa. ${ }^{14}$

In a clinical case scenario of the correct antibiotic use for methicillin-sensitive Staphylococcus aureus, only $29.4 \%$ of interns knew what antibiotics to prescribe. A reason for this low score could be the fact that the question was not read carefully. The term 'methicillin-resistant' is usually used when referring to ABR. Thus, the term 'methicillin-sensitive' may have been misread. It should be noted, however, that this correlates with the fact that only $54.4 \%$ of respondents felt adequately prepared to prescribe antibiotics.

In the clinical case scenario, the correct management of upper respiratory tract infection was reported by $62.0 \%$ of interns. This percentage is lower than expected because upper respiratory tract infections are commonly seen, ${ }^{15}$ and it would be expected that most interns would know how to manage it correctly. Most upper respiratory tract infections are caused by viral infections where antibiotic prescribing will have no effect. Research has demonstrated high rates of unnecessary antibiotic prescribing for these types of infections, which promotes ABR..$^{15}$ In order to promote antibiotic stewardship, better management of upper respiratory tract infections is needed.

The correct management of a drip site infection was only identified by $4.4 \%$ of the interns. Serious intervention is needed to improve the management of drip site infections as it is a fairly common occurrence in hospital environments. ${ }^{16}$

Less than half of the respondents $(47.8 \%)$ knew how to correctly assess the severity and management of Clostridium difficile infection. Better management of the infection is needed as Clostridium difficile is the number one causative agent of antibiotic-associated and healthcare-associated infective diarrhoea in hospital environments. ${ }^{16}$

The correct antimicrobial selection for ESBL-positive Escherichia coli bacteraemia was made by $18.5 \%$ of interns. As extended spectrum infections are becoming more common as 
a result of $\mathrm{ABR},{ }^{11}$ correct treatment is needed to contain $\mathrm{ABR}$. Overall, knowledge about antibiotic prescribing regimens and $A B R$ are moderate to poor, which correlates with the South African final-year medical student study. ${ }^{11}$

The responses to the clinical case scenarios in the study questionnaire showed that some interns struggle to choose the right antibiotic treatment although $55.4 \%$ indicated that they felt prepared to choose the correct antibiotic.

\section{Perceptions}

The majority of intern medical doctors (85.9\%) in this study realised that $A B R$ is a problem in South Africa, but only $63.0 \%$ perceived ABR as a problem in hospitals where they have rotated. Studies have shown that a large number of medical students or doctors are ignorant to the fact that ABR is a problem in their immediate environment and this may contribute to over-prescription. ${ }^{11,17}$

Nearly a fifth of the respondents (19.6\%) disagreed that the unnecessary use of broad-spectrum antibiotics increases $A B R$. As one of the main factors of increased $A B R$ is the unnecessary use of antibiotics, interns need to become more aware about the causes of ABR. It is encouraging to see that the majority (89.1\%) of interns indicated that they would like more education on ABR.

In our study, interns indicated that they lack training and preparedness in certain areas of antibiotic prescribing. This finding is in accordance with the study done on final-year medical students in South Africa. ${ }^{11}$ In a study done on junior doctors in London, ${ }^{12}$ less than 15\% of participants indicated that their previous antimicrobial education was effective, and $60 \%$ of participants who had qualified the previous year stated that they required further education in antimicrobial prescribing. This latter percentage was $74.0 \%$ for more experienced junior doctors. Specific topics identified for further education in that study were: '(i) principles of antimicrobial prescribing, (ii) diagnosis of infections, (iii) clinical review of patients with infections, (iv) prescribing in the context of antimicrobial resistance and (v) laboratory testing and test results' (p. 9). ${ }^{12}$

Three quarters of participating interns attended lectures or tutorials on the dosing duration of antibiotic treatment for specific infections. However, less than half of interns felt like they were prepared when it comes to the dosing duration of antibiotics. This gap can possibly be attributed to ineffective educational methods, too little time spent on preparing interns for dosing duration or intern medical doctors not frequently educated on the subject throughout their undergraduate training and career.

Half of the interns felt very prepared on how and when to transition from intravenous to oral antibiotic treatment compared to nearly $80 \%$ of the interns who reported to have had education on the indications of intravenous antibiotics. The disparity between education and knowledge is once again apparent.

Only 59.8\% of interns have a perceived high understanding of the basic mechanisms of ABR; a fact that is troubling because understanding what causes resistance is an important way to curb ABR. When interns do not know that some of their actions can promote ABR, they will continue to unintentionally contribute to the problem. The fact that only $50.0 \%$ interns agreed that poor hand hygiene to $\mathrm{ABR}$ is concerning. This correlates with the final-year medical student study. ${ }^{11}$

\section{Education}

Interns' responses to the various available education's effectiveness give an indication of what they would prefer. The majority reported that formal lectures, formal bedside tutorials, and consultant ward rounds are useful for learning about antibiotic spectrums, resistance and prescribing. Areas where intern medical doctors felt the least prepared were an interpretation of antibiograms, knowledge of dosing duration and performing de-escalation in antibiotic therapy. In the study done on final-year medical students, these were also the areas in which students felt the least prepared. ${ }^{11}$

Interns' suggestions regarding useful approaches to prepare them for prescribing antibiotics place emphasis on alternative approaches rather than formal classes. An Australian study on junior doctors found that an educational session in conjunction with pocket guidelines improved self-confidence regarding vancomycin prescribing and monitoring. ${ }^{18}$

\section{Study strengths and limitations}

The study had a high response rate and made use of an existing questionnaire that enabled direct comparison with the recent South African study on final-year medical students. Whilst evaluating the theoretical knowledge of antibiotic dosing and duration, it should be acknowledged that interns in the clinical setting make use of resource material such as the Internet to guide them. This could indicate that they would fare better in practice than shown in this study.

\section{Recommendations}

For antibiotic stewardship programmes to improve, attention must be given to each aspect of antibiotic stewardship. More attention must be given to choosing the correct antibiotics and dosage, via the correct application route for the right amount of time, as well as better infection control. Better inpatient care practices should be taught and applied so that hand hygiene is not one of the leading causes of ABR.

There is a definite gap between undergraduate training, theoretical knowledge and clinical knowledge in intern medical doctors. Certain areas of medical value should be emphasised more. Changes need to be made in the educational method in which interns learn about starting 
antibiotic treatment and the dosing duration of antibiotic therapy. Ward rounds can focus more on the indications for intravenous antibiotic treatment and when to transfer to other application routes.

Intern medical doctors should be made more aware of the basic mechanisms of $\mathrm{ABR}$, as this study has proven that most interns admit to not knowing the fundamental principles of $\mathrm{ABR}$. This can be achieved through training during weekly meetings or training during medical school.

All doctors should be made aware, preferably at an early stage during medical school training, that ABR is a problem in their immediate environment to promote the applicability of antibiotic stewardship programmes. Small steps can be taken to improve antibiotic stewardship programmes such as promoting the practice of proper hand hygiene routines.

Educational methods in the medical curriculum should be adapted according to indications of what interns and finalyear medical students would prefer.

Future research should investigate factors contributing to poor antibiotic prescribing knowledge amongst intern medical doctors.

\section{Conclusion}

Our results show that intern medical doctors do not have basic antibiotic prescribing knowledge, but there is a desire for more education in this area. Provision of more information and adapted teaching methods for interns may contribute to a reduction in the prevalence of $\mathrm{ABR}$ and promote antibiotic stewardship.

\section{Acknowledgements}

The authors would like to thank the Research Committee of the School of Medicine, University of the Free State, for supplying the funding necessary to complete this study and Ms. T. Mulder, medical editor/writer, Faculty of Health Sciences, UFS, for editorial and technical preparation of the manuscript.

\section{Competing interests}

The authors declare that they have no financial or personal relationships that may have inappropriately influenced them in writing this article.

\section{Authors' contributions}

A.A., M.B., R.d.L. and E.J.v.V. developed the protocol, performed the data collection, and did the initial write up of this study. S.P. assisted with protocol development. T.E.K. was the supervisor of this study, suggested the concept, assisted with the protocol development, data collection and interpretation of data and write up of this study. G.J. assisted with the planning, performed data analysis, and assisted with the interpretation and write up of the article.

\section{Funding information}

Funding was received from the Research Committee of the School of Medicine, UFS.

\section{Data availability}

The data that support the findings of this study are available from the corresponding author, T.E.K., upon reasonable request.

\section{Disclaimer}

The views and opinions expressed in this article are those of the authors and do not necessarily reflect the official policy of any affiliated agency of the authors.

\section{References}

1. World Health Organization. Antimicrobial resistance: Global report on surveillance 2014 [homepage on the Internet]. Geneva: World Health Organization; 2014 [cited 2017 Mar 13]. Available from: http://www.who.int/drugresistance/ documents/surveillancereport

2. Dryden MS, Cooke J, Davey P. Antibiotic stewardship - More education and regulation not more availability? J Antimicrob Chemother. 2009;64(5):885-888. https://doi.org/10.1093/jac/dkp305

3. World Health Organization. Antibiotic resistance [homepage on the Internet] Geneva: World Health Organization; 2020 [cited 2021 Aug 10]. Available from: http://www.who.int/news-room/fact-sheets/detail/antibiotic-resistance

4. World Health Organization. Antimicrobial resistance [homepage on the Internet] Geneva: World Health Organization; 2020 [cited 2021 Aug 10]. Available from: https://www.who.int/news-room/fact-sheets/detail/antimicrobial-resistance

5. Lesprit P, Brun-Buisson C. Hospital antibiotic stewardship. Curr Opin Infect Dis. 2008;21(4):344-349. https://doi.org/10.1097/QCO.0b013e3283013959

6. Mendelson M. Role of antibiotic stewardship in extending the age of modern medicine. S Afr Med J. 2015;105(5):414-418. https://doi.org/10.7196/samj.9635

7. OhI CA, Luther VP. Health care provider education as a tool to enhance antibiotic stewardship practices. Infect Dis Clin North Am. 2014;28(2):177-193. https://doi. org/10.1016/j.idc.2014.02.001

8. Brink AJ, Messina AP, Feldman C, et al. Antimicrobial stewardship across 47 South African hospitals: An implementation study. Lancet Infect Dis. 2016;16(9): 1017-1025. https://doi.org/10.1016/S1473-3099(16)30012-3

9. Dyar OJ, Nathwani D, Monnet DL, et al. Do medical students feel prepared to prescribe antibiotics responsibly? Results from a cross-sectional survey in 29 European countries. J Antimicrob Chemother. 2018;73(8):2236-2242. https://doi. org/10.1093/jac/dky150

10. Weier $\mathrm{N}$, Thursky K, Zaidi STR. Antimicrobial knowledge and confidence amongst final year medical students in Australia. PLoS One. 2017;12(8):e0182460. https:// doi.org/10.1371/journal.pone.0182460

11. Wasserman S, Potgieter S, Shoul E, et al. South African medical students perceptions and knowledge about antibiotic resistance and appropriate prescribing: Are we providing adequate training to future prescribers? S Afr Med J. 2017;107(5):405-410. https://doi.org/10.7196/SAMJ.2017.v107i5.12370

12. Gharbi M, Moore LS, Castro-Sánchez E, et al. A needs assessment study for optimising prescribing practice in secondary care junior doctors: The Antibiotic Prescribing Education among Doctors (APED). BMC Infect Dis. 2016;16(1):456. https://doi.org/10.1186/s12879-016-1800-z

13. Mattick K, Kelly N, Rees C. A window into the lives of junior doctors: Narrative interviews exploring antimicrobial prescribing experiences. J Antimicrob Chemother. 2014;69(8):2274-2283. https://doi.org/10.1093/jac/dku093

14. Madhi SA, Petersen K, Madhi A, Khoosal M, Klugman KP. Increased disease burden and antibiotic resistance of bacteria causing severe community-acquired lowe respiratory tract infections in human immunodeficiency virus type 1-infected children. Clin Infect Dis. 2000;31(1):170-176. https://doi.org/10.1086/313925

15. Bagger K, Nielsen AB, Siersma V, Bjerrum L. Inappropriate antibiotic prescribing and demand for antibiotics in patients with upper respiratory tract infections is hardly different in female versus male patients as seen in primary care. Eur J Gen Pract. 2015;21(2):118-123. https://doi.org/10.3109/13814788.2014. 1001361

16. Nazarko L. Understanding and managing Clostridium difficile. Nurs Residentia Care. 2009;11(10):490-495. https://doi.org/10.12968/nrec.2009.11.10.44020

17. Abbo LM, Cosgrove SE, Pottinger PS, et al. Medical students' perceptions and knowledge about antimicrobial stewardship: How are we educating our future prescribers? Clin Infect Dis. 2013;57(5):631-638. https://doi.org/10.1093/cid/cit370

18. Phillips CJ, McKinnon RA, Woodman RJ, Gordon DL. Junior doctors' preparedness to prescribe, monitor, and treat patients with the antibiotic vancomycin in an Australian teaching hospital. J Educ Eval Health Prof. 2017;14:13. https://doi. org/10.3352/jeehp.2017.14.13 